\title{
Oxidant killing of the malaria parasites
}

\section{from F.E.G. Cox}

THE continuing existence and spread of malaria coupled with an increasing incidence of drug resistance has encouraged those working in both immunology and chemotherapy to ask what exactly it is that kills malaria parasites in red blood cells. Clues and confusion abound but in the case of the rodent malaria parasites most widely used as models for human malaria, antibody seems to have little effect on Plasmodium vinckei and $P$. chabaudi that inhabit mature red blood cells whereas in infections with $P$. berghei and $P$. yoelii that preferentially invade reticulocytes antibody probably does play a part in parasite killing ${ }^{1}$. The involvement of nonspecific factors in the killing of $P$. vinckei and $P$. chabaudi has been mooted for several years and the possibility that these factors are macrophage products has been championed by an Australian scientist, Ian Clark, and his colleagues ${ }^{2}$. At the Fifth International Congress of Parasitology held in Toronto in August, Clark presented new data implicating toxic oxygen radicals in the killing of malaria parasties and the experimental evidence has just been published ${ }^{3,4}$ and supported by parallel studies in other laboratories 5,6 .

Clark's approach has been direct. In his first series of experiments he injected alloxan, which generates reactive forms of oxygen as a result of redox cycling between alloxan and dialuric acid, into mice infected with $P$. vinckei and found that the parasitaemias fell from $30-60$ per cent to less than 10 per cent within an hour and then continued to fall to very low levels after which further injections cured the normally lethal infections completely ${ }^{3}$. The decline in parasitaemia was not due to any diabetogenic effects of alloxan as previous injection of glucose did not reverse the effect whereas pretreatment with desferriozamine a specific iron chelator that inhibits the formation of hydroxyl radicals, did. Similar, but less dramatic, effects were obtained with hydrogen peroxide and phenylhydrazine. In all the experiments, cure was accompanied by transient haemolysis and the intraerythrocytic death of the parasites. Organic hydroperoxides decompose to produce oxygen or peroxide radicals directly and in his second series of experiments Clark injected $t$-butyl hydroperoxide into mice infected with $P$. vinckei with results substantially similar to those obtained using alloxan ${ }^{4}$. Allison, using very small numbers of animals, also found that repeated doses of $t$-butyl hydroperoxide had some adverse effects on another rodent malaria parasite, $P$. yoelii, when the initial parasitaemias were low but very little effect when at the levels used for $P$. vinckei in the experiments described above ${ }^{6}$.

Hydrogen peroxide has been implicated as a possible mechanism of malaria parasite killing by another group of scientists at the Middlesex Hospital Medical School in London ${ }^{5}$. In these experiments, four parasites were used $-P$. chabaudi, an avirulent line of $P$. yoelii, a virulent and lethal line of $P$. yoelii and $P$. berghei. The injection of hydrogen peroxide into infected mice rapidly reduced the parasitaemias in all cases except $P$. berghei. In in vitro experiments, hydrogen peroxide was found to kill the two parasites investigated - the avirulent $P$. yoelii and $P$. berghei equally effectively.

These examples taken together indicate that oxygen or peroxide radicals or other derivatives of oxygen are involved in the killing of $P$. vinckei, $P$. chabaudi and a lethal line of $P$. yoelii (which invades mature erythrocytes) but not $P$. berghei. Whether this is due to the type of red cell invaded or to intrinsic factors in the parasites is not at all clear.

These experiments represent very artificial situations but if oxygen-mediated killing occurs during naturally resolving infections or after vaccination, what cells produce the reactive substances? Both the Australian $^{3}$ and London ${ }^{5}$ scientists suggest that these are macrophages and that as infected blood cells squeeze between the fixed macrophages of the spleen and liver they trigger oxidative bursts and are subjected to oxygen products released by these cells. Evidence in support of this idea comes from other work with $P$. berghei in which it has been shown that peritoneal macrophages react with a chemiluminescent response, indicative of the generation of activated oxygen species, in the presence of whole or lysed parasitized erythrocytes? On the other hand, during $P$. berghei infections in rats, the activity of superoxide dismutase (which removes superoxide) is reduced in the liver but normal elsewhere while catalase activity responsible for the breakdown of hydrogen peroxide is reduced in the liver but increased in the spleen $^{8}$. The reduction of superoxide dismutase levels would lead to raised levels of oxygen radicals in the liver without the need to invoke any increased activity or parasite triggering. In the context of these experiments it is probably too simplistic to attribute comparable roles to the liver and spleen. It has also been suggested that cer- tain natural killer cells may be the effector cells ${ }^{6}$ but no direct evidence has been offered to support this.

Although all these experiments relate to rodent malarias there is every indication that the same or similar procesess may damage human parasites as $t$-butyl hydroperoxide inhibits the development of $P$. falciparum in culture ${ }^{4}$. If this is the case then there are a number of implications there is a mass of evidence that shows that oxygen radicals are toxic ${ }^{9}$ and kill protozoa $^{10}$ and malaria now joins the list; the pathology of malaria is commensurate with the production of toxic oxygen products ${ }^{3}$ or with a loss of superoxide dismutase ${ }^{8}$, and the possibility that infected cells are under oxygen stress and therefore more susceptible to the effects of oxygen radicals could explain the adaptive advantage of mutations, such as sickle cell trait, that allow certain individuals to resist malaria ${ }^{3,6}$; and finally, it is possible that antimalarial drugs could operate by releasing oxygen radicals and could thus lead to a rational approach to the design of new drugs ${ }^{4}$. That immunity and chemotherapy should go hand in hand has always been a dream of parasitologists and there is every sign that this could now become a reality.

Caution must of course be paramount and it is a long way from mouse to man and we do not even know which species of rodent parasite most closely resembles the human form. The intimate relationship between protection and pathology should also provide grounds for concern. There will be many more studies in this exciting area and it will be some time before we know whether these recent experiments represent a solution or even a clue to the riddle of malaria parasite killing or whether they simply add to the confusion.

1. Allison, A.C. Fortschr. Zool. 27,245 (1982)

2. Clark, I.A. et al. Infect. Immun. 32, 1058 (1981)

3. Clark, I.A. \& Hunt, N.H. Infect. Immun. 39, 1 (1983).

4. Clark, I.A. et al. Lancel, I, 234 (1983).

5. Dockrell, H.M. \& Playfair, J.H.L. Infect. Immun. 39, 456 (1983).

6. Allison, A.C. \& Eugui, E.M. Lancet ii, 1431 (1982)

7. Makimura, S. et al.. Infect. Immun. 37, 800 (1982)

8. Kulkarni, A. B. et al. Bull. Haffkine Inst. 9, 29, (1981)

9. Halliwell, B. Trends biochem. Sci. August. 270 (1982) 10. Maul, J. Fortschr. Zool. 27, 43 (1982).

\section{ERRATUM}

In the article 'Increasing atmospheric methane' (301, 568; 17 February, 1983) the first eleven lines of the second column were accidentally transposed to the top of the third column. The section should have read:

The temperature rise is roughly 38 per cent of the increase calculated to have occurred as a result of increases in atmospheric $\mathrm{CO}_{2}$. Furthermore, since methane plays a central role in the chemistry of the atmosphere the increase may have caused changes in the level of other atmospheric constituents such as $\mathrm{OH}, \mathrm{CO}$ and $\mathrm{O}_{3}$.

How can we explain the methane trends sug gested by the data of Craig and Chou? What process or processes could have caused methane suddenly to increase towards the end of the sixteenth century after remaining constant for at least 25,000 years?

We apologise to the author, W.L. Chameides. 